\title{
Intermittency between avalanche regimes on grain piles
}

\author{
Arran, M.I.* and Vriend, N.M. \\ Department of Applied Mathematics and Theoretical Physics, \\ University of Cambridge, Wilberforce Road, \\ Cambridge, CB3 0WA, United Kingdom
}

\begin{abstract}
We experimentally investigate discrete avalanches of grains, driven by a low inflow rate, on an erodible pile in a channel. We observe intermittency between one regime, in which avalanches are quasi-periodic and system-spanning, and another, in which they pass at irregular intervals and have a power-law size distribution. Observations are robust to changes of inflow rate and grain type, and require no tuning of external parameters. We demonstrate that the state of the pile's surface determines whether avalanche fronts propagate to the end of the channel or stop partway down, and we introduce a toy model for the latter case that reproduces the observed power-law size distribution. We suggest direct applications to avalanches of pharmaceutical and geophysical grains, and the possibility of reconciling the 'self-organized criticality' predicted by several authors with the hysteretic behavior described by others.
\end{abstract}


Discrete avalanches on the surface of grain piles appear in contexts as diverse as desert sand dunes [1, 2] and agricultural corn silos [3, 4]. The size distributions of such avalanches are significant in a number of these contexts, controlling the assessed 'flowability' of pharmaceutical grains [5], and determining the hazard posed by rock falls above talus slopes [6].

The topic has attracted interest from the wider physics community as a result of Bak, Tang and Wiesenfeld's 'sandpile' model, a cellular automaton which introduced the concept of self-organized criticality. This concept has been invoked in areas from solar flares [7] through forest fires [8] to neuron firing [9], but conditions for its applicability remain unclear $[10,11]$. If applied to physical piles of grains, for example, the 'sandpile' model predicts a power-law distribution of avalanche sizes [12-14], whereas such distributions have only been observed for grains of specific varieties of rice [15], and for grain rearrangements long before avalanching [16, 17]. Most authors have instead reported quasi-periodic, system-spanning avalanches, either in rotating drums [18-21] or on the surface of a short pile [15, 22].

Those authors reporting quasi-periodic avalanches explain the limited applicability of the 'sandpile' model to physical piles of grains by the model's omission of inertial effects, with the model's avalanches stopping as soon as the condition for their initiation is no longer satisfied. Physical avalanches are inertial, so that, under the addition of grains, a static grain pile is metastable whenever its slope is between an angle of repose $\theta_{r}$, at which any avalanche will stop, and a maximum slope angle $\theta_{m}$, at which any disturbance initiates an avalanche [23-25]. The associated hysteresis between static and flowing grains is associated with a time interval between avalanches, during which the top of the pile steepens from $\theta_{r}$ to $\theta_{m}$. Approaching $\theta_{m}$, the pile yields and an avalanche propagates downslope, leaving behind it a slope at $\theta_{r}$ on which future avalanches will likewise continue to propagate. This physical description explains the quasi-periodic, system-spanning avalanches hitherto observed, and the matter has been considered settled.

In this paper we report entirely new behavior: without tuning of external parameters, a grain pile in a long channel exhibits intermittency between two regimes. In one regime, avalanches are quasi-periodic and system-spanning, as previously reported. In the other, the time intervals between avalanches are irregular and most avalanches stop partway down the channel, with the frequency distribution of their lengths satisfying a power-law. We successfully reproduce behavior in this new regime with a toy model, in which the power- 
law size distribution arises from interactions between each avalanche and the stopped fronts of previous avalanches. We show furthermore that the existence of the two regimes, and of the spontaneous transitions between them, can be qualitatively described by a reduced dynamical system. Our experiments involve inertial avalanches of $O\left(10^{6}\right)$ grains, and our observations are robust to changes of inflow rate and of grain type, demonstrating their relevance to a variety of grain piles.

For our experiments, we first use angular construction sand with mean particle diameter $\bar{d}=0.47 \mathrm{~mm}$, standard deviation in particle diameter $0.10 \mathrm{~mm}$, and angle of repose $\theta_{r}=32^{\circ}$. An $L=2.5 \mathrm{~m}$ long channel of width $W=5 \mathrm{~cm}$ is inclined at $\theta_{r}$, as depicted in Fig. 1 . Grains flow into the channel via a nozzle at its top, while its bottom is partially closed by an inclined plate, establishing an $11 \mathrm{~cm}$ deep pile of static grains at their angle of repose. We then permit further grains to flow through the nozzle with constant flux $Q=3.05 \mathrm{~cm}^{3} \mathrm{~s}^{-1}$. This is sufficiently small that the pile increases in height slowly and locally at the channel's top before losing stability, so that discrete avalanches flow along the channel on the surface of the pile, with a quiescent interval between each avalanche and the next. Footage of avalanching is included as Supplementary Material [26].

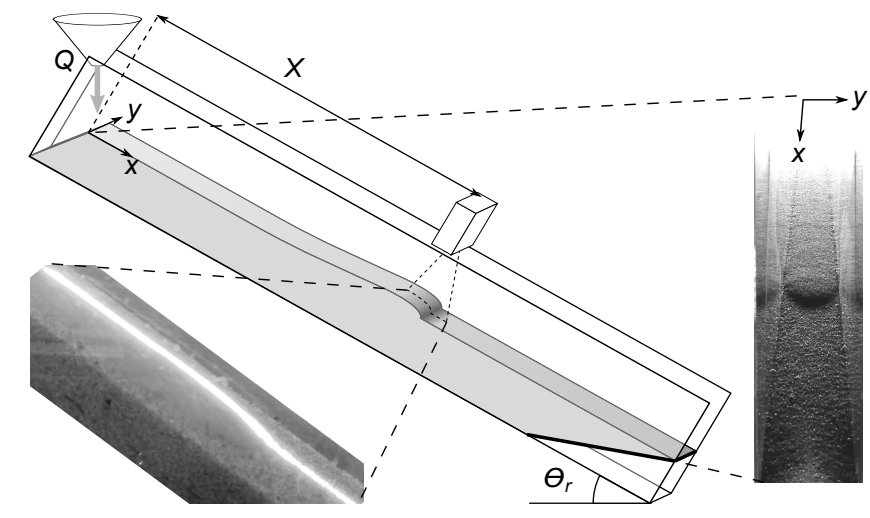

FIG. 1: Schematic of experimental apparatus. The channel is smooth-walled and has a base roughened with sandpaper. Inset, right, is an image of an avalanche propagating down the channel on the surface of the grain pile, while inset, left, is an image of such an avalanche's front in the field of view of a laser profile scanner.

In each experiment, a Micro-Epsilon scanCONTROL 2800-100 laser profile scanner measures surface height along a section of the channel's centerline, at a fixed distance $X$ downslope. From these height profiles, we may distinguish the times at which avalanches pass the scanner. While maintaining identical inflow conditions, we observe alternation between 


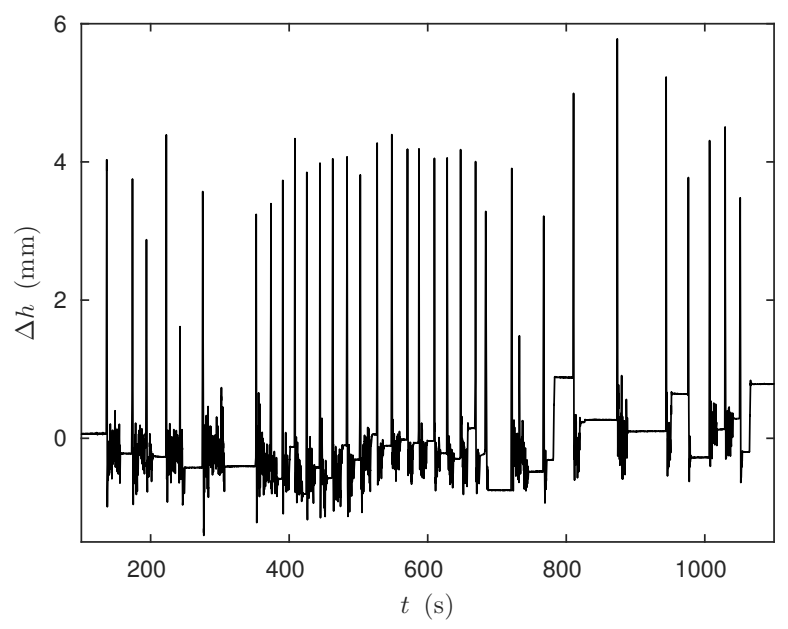

FIG. 2: Alternation between quasi-periodic and irregular regimes. When fixed a distance $X$ downslope, the scanner measures surface heights $h\left(x_{i}\right)$ at distances $x_{i}$ downslope, with $X$ the midpoint of $x_{i}$. We calculate $\Delta h=\left\langle h\left(x_{i}\right)\right\rangle_{x_{i}<X}-\left\langle h\left(x_{i}\right)\right\rangle_{x_{i}>X}$. Over time $t$, each avalanche front passing the scanner is associated with a peak in $\Delta h$. At a distance $X=0.34 \mathrm{~L}=844 \mathrm{~mm}$ downslope, avalanches pass quasi-periodically between times $t=350 \mathrm{~s}$ and $t=680 \mathrm{~s}$, while at earlier and later times the intervals between avalanches are irregular.

two regimes: one in which avalanches pass the scanner quasi-periodically (henceforth 'the quasi-periodic regime') and another in which the intervals between avalanches are irregular (henceforth 'the irregular regime'). An example of this alternation is depicted in Fig. 2.

Since all avalanches propagate from the channel's top, the avalanches passing the scanner are those with a length greater than $X$. We measure the time intervals between such avalanches using the data processing described in the Supplementary Material, classify in the quasi-periodic regime any 12 consecutive intervals with a standard deviation less than $20 \%$ of their mean, and classify in the irregular regime any intervals that are not in a consecutive set of 6 with a standard deviation less than $20 \%$ of their mean. For each regime, we calculate the mean of the intervals in that regime, $\bar{T}(X)$. Supposing an avalanche to involve some minimum number of grains, we expect this mean interval to scale with $\bar{d}^{3} / Q$.

To find the size distribution of avalanches in each regime, we change the position of the scanner, varying $X$. We observe that in the quasi-periodic regime $\bar{T}$ remains constant (Fig. 3, $R^{2}=0.011, p$-value for rejection 0.5, as described in the Supplementary Material). This indicates that each avalanche propagates the entire length of the channel and flows out over the inclined plate at its end, in accord with the reports of Ref.s [15, 18-22] and corresponding to the hysteresis between static and flowing grains that they and we have described. In the 


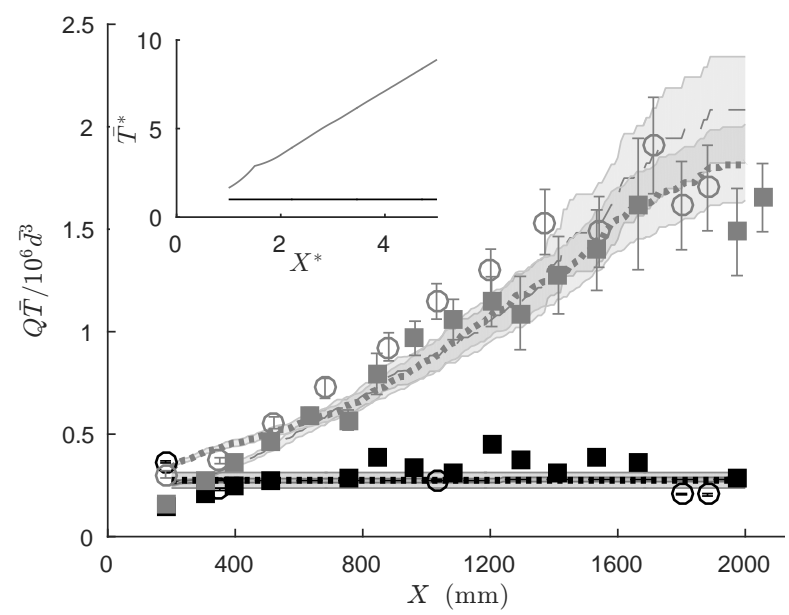

FIG. 3: Hysteretic and power-law behavior in the quasi-periodic (black) and irregular (gray) regimes. We conduct experiments with continuous fluxes $Q=0.9$ (०) and $3.05(-) \mathrm{cm}^{3} \mathrm{~s}^{-1}$ of sand $(\bar{d}=0.47 \mathrm{~mm})$; as well as with interrupted inflows of the same sand (- ) and of glass beads $(\cdots, \bar{d}=0.22 \mathrm{~mm})$. Error bars and shaded regions represent the standard errors of the means over observed avalanches. For $X>2000 \mathrm{~mm}$, the effect of the channel's end is significant. Raw data is in the Supplementary Material. We model the quasi-periodic regime with the assumption that all avalanches propagate to the end of the channel, while an overrunning-mobilization model reproduces the behavior observed in the irregular regime (inset).

irregular regime, however, $\bar{T}$ increases linearly with $X\left(R^{2}=0.94, p\right.$-value for rejection 0.59). The frequency of avalanches which have a length greater than $X$ therefore decays as $X^{-1}$, and hence the probability density of an avalanche being of length $X$ decays as $X^{-2}$.

We repeat these experiments with a smaller inflow nozzle, decreasing the flow rate $Q$ by a factor of more than 3 , to $0.9 \mathrm{~cm}^{3} \mathrm{~s}^{-1}$. Scaling times $\bar{T}$ by $Q$ we observe excellent collapse, indicating a separation of timescales between the slow forcing, via input of gravitational potential energy, and the fast avalanching. This demonstrates that our experiments are in the low- $Q$ limit that is relevant to many physical situations and is a precondition for the applicability of self-organized criticality. Since our system also features both non-linear interactions and avalanching, and the observed power-law decay is among self-organized criticality's hallmarks $[10,11,27]$, it is reasonable to compare our results to others for which the existence of self-organized criticality is claimed.

We seek to explain the size distribution of avalanches in the irregular regime, in which most avalanches stop after propagating only a short distance. In this regime, if an avalanche propagates sufficiently far down the channel to overrun the stopped front of a previous avalanche, it mobilizes the static grains, resulting in a larger avalanche that is able to prop- 
agate further. To model this process, we first assume that avalanching is instantaneous in comparison to the intervals of time $T_{i}(0)$ between one avalanche and the next, which we take to be independent and identically distributed, with mean $\tau$. We then make the simplifying assumptions that stopped avalanche fronts are of equal height $H$, and that each front leaves behind it a slope at the angle of repose. In the absence of stopped avalanche fronts, the $i$ th avalanche would therefore propagate a distance $x_{i}^{0}=Q T_{i}(0) / H W$. Furthermore, each stopped avalanche will, if its front is overrun, add its own length to the final length of the overrunning avalanche. We may write the process algorithmically, with at each time the distances downslope of stopped avalanche fronts denoted by the increasing sequence $S=\left(s_{1}, s_{2}, \ldots, s_{n}\right)$. Iterating over avalanche number $i$ :

1. Initialize avalanche length $x_{i} \mapsto x_{i}^{0}$ (the slope yields, mobilizing a given volume of grains)

2. While $x_{i} \geq s_{1}$ (the avalanche is able to overrun the first stopped avalanche front), $S \mapsto\left(s_{2}, s_{3}, \ldots, s_{n}\right)$ and $x_{i} \mapsto x_{i}+s_{1}$ (the stopped avalanche is mobilized, adding its length to that of the overrunning avalanche).

3. Once $S=\varnothing$ or $x_{i}<s_{1}$ (no stopped front can be overrun), $S \mapsto\left(x_{i}, S\right)$ (the avalanche stops a distance $x_{i}$ downslope).

Simulating this process numerically, with $\tau=H=W=Q=1$ and with the distribution of $T_{i}(0)$ taken in turn to be uniform, lognormal and exponential, we find that this model system reproduces the linear increase with $X$ of $\bar{T}(X)$ (see inset, Fig. 3, for which time intervals $T_{i}(0)$ are drawn from a uniform distribution between 0.5 and 1.5). The asymptotic behavior of $\bar{T}$ at high $X$ is insensitive to the distribution of $T_{i}(0)$.

To determine the relevance of this overrunning-mobilization model, we repeat the above experiments, but now halt inflow upon the start of each avalanche. After the avalanche has come to a stop, surface height is measured over the entirety of the channel's centerline, and the position of the stopped avalanche front is detected from this full-length height profile (details of detection are described in the Supplementary Material). Inflow is then resumed and the time interval until the start of the next avalanche recorded, with this process repeated over 400 avalanches. As no grains flow into the channel during each avalanche, this procedure is equivalent to conducting experiments with an infinite separation of timescales, and we observe in Fig. 3 the same behavior of $\bar{T}$ as in the original experiments. 
The new data support the relevance of the overrunning-mobilization model. Considering the interaction of flowing avalanches with stopped avalanche fronts, we observe in Fig. 4 the same qualitative behavior in experiments as is predicted by the model. However, in experiments, the exact position at which an avalanche stops is sensitive to the state of the static pile over which it propagates, as demonstrated by the early cessation of the avalanches depicted in Fig. 4a at $t=111 \mathrm{~s}$ and $t=123 \mathrm{~s}$. This sensitivity prevents us from accurately predicting, at this time, which stopped avalanche fronts will be overrun by a given avalanche, and hence from accurately predicting the final length of individual avalanches.

Instead, we calculate the correlation between observed avalanche lengths and the lengths predicted by the overrunning-mobilization model, over a large number of avalanches. We consider the last 200 avalanches in the irregular regime, for which our measurements of the time intervals between avalanches are most precise, and for each of the preceding 10 avalanches calculate a value $H_{i}$ equal to the mean increase in surface height in the wake of the avalanche's stopped front, with respect to the last prior height profile with no stopped avalanche fronts in the corresponding region. To predict avalanche lengths, we take $H$ to be the median of our measured values $H_{i}$, we use our measured values of $Q$ and $W$, and we input to the overrunning-mobilization model the positions of stopped avalanche fronts prior to each avalanche and the time interval $T_{i}(0)$ before each avalanche. Pearson's correlation coefficient between our predictions and our observations is 0.45 . While this value indicates that effects other than overrunning and mobilization are important, the correlation is highly significant; the probability of such correlation would be $p=10^{-11}$ if avalanche lengths were randomly sampled from the distribution of their lengths.

Having described behavior in each of the two regimes, we use the full-length height profiles to analyze the intermittency between them. We consider the state of the static grain pile after each avalanche that propagates to the end of the channel, which we refer to as a 'zero-front state'. In particular, we consider two statistics: the average slope angle $\bar{\theta}$, as determined by the gradient of the least-squares line of best fit to each full-length height profile; and the variance $\sigma^{2}$ of residuals from that line of best fit. The higher the slope variability $\sigma^{2}$, the more regions of shallow local slope the subsequent avalanche will have to pass over, making it more likely to stop. The higher the value of $\bar{\theta}$, the more potential energy will be available to the subsequent avalanche, making it more likely to overcome those regions and hence less likely to stop. 

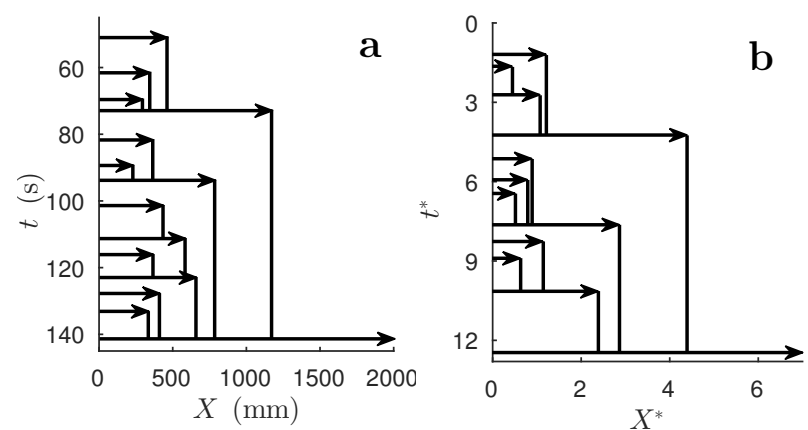

FIG. 4: Comparison of experimentally observed avalanche-front interactions with realizations of the overrunning-mobilization model. Avalanches paths as observed in experiments (a) and simulations (b) appear as horizontal arrows down the channel, while stopped avalanche fronts appear as vertical lines with constant $X$. In $\mathbf{b}$ we use a log-normal distribution of nondimensionalized intervals between avalanches, with mean 1 and standard deviation $1 / 2$.

In Fig. 5a, a trajectory in the $\sigma^{2}-\bar{\theta}$ plane elucidates the mechanism for intermittency. $\sigma^{2}$ is low in zero-front states subsequent to quasi-periodic, system-spanning avalanches, making the subsequent avalanche unlikely to stop, and maintaining the system in the same regime. However, such avalanches cause net erosion of grains at the channel's top, decreasing $\bar{\theta}$ over time. At some point an avalanche will have insufficient potential energy to overcome even minor regions of shallow local slope, stopping partway down the slope, and forcing the system into the irregular regime, despite the value of $\sigma^{2}$. In zero-front states subsequent to irregular avalanches, $\sigma^{2}$ is high, so that most avalanches continue to stop partway down the slope even after each of the rare avalanches that propagate to the end of the channel. However, the erosion and deposition of grains are highly sensitive to the details of stopped avalanche fronts' emplacement and mobilization, resulting in large changes in both $\sigma^{2}$ and $\bar{\theta}$ over consecutive zero-front states. At some point, the subsequent avalanche has sufficient available potential energy to propagate to the channel's end, forcing the regime into the quasi-periodic regime.

Figure $5 \mathrm{~b}$ provides further evidence for this mechanism, demonstrating the change in surface height at the top of the channel, a proxy for $\bar{\theta}$, over the course of over 2000 avalanches. Furthermore, changing $\bar{\theta}$ directly in the course of an experiment, by raising or lowering the entire channel's inclination by as little as $0.1^{\circ}$, forces the system from the irregular regime to the quasi-periodic regime, or vice versa. Directly increasing $\sigma^{2}$, by lightly brushing the surface of the grain pile, forces the system from the quasi-periodic regime to the irregular 

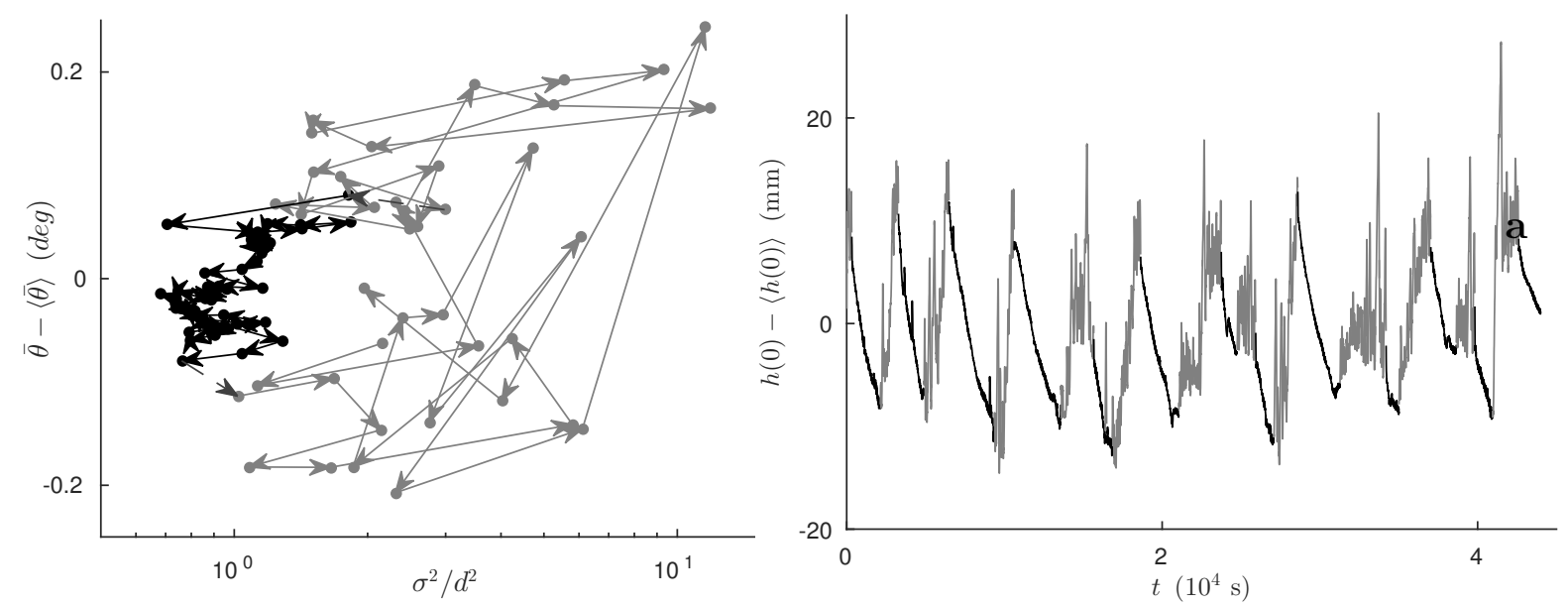

FIG. 5: System evolution, in the $\sigma^{2}-\bar{\theta}$ plane over one cycle between regimes (a), and in $h(0)$ over multiple cycles (b). The slope variability $\sigma^{2}$ remains low while the system is quasi-periodic (black) and high while it is irregular (gray), maintaining each regime. The average slope angle $\bar{\theta}$, meanwhile, provides the stimulus for changes of regime, varying chaotically in the irregular regime and recovering gradually in the quasi-periodic

regime. Surface height at the top of the channel $h(0)$, smoothed over individual avalanches, is a proxy for $\bar{\theta}$.

regime.

To verify the applicability of these results to other types of grains, we replace sand in our experiments with an inflow flux $Q=1.22 \mathrm{~cm}^{3} \mathrm{~s}^{-1}$ of spherical glass beads, halted during each avalanche. Previous authors have shown avalanches of such grains to be substantially different from those of sand $[28,29]$. The glass beads have mean particle diameter $\bar{d}=$ $0.22 \mathrm{~mm}$, standard deviation in particle diameter $0.07 \mathrm{~mm}$, and angle of repose $\theta_{r}=25^{\circ}$. Avalanches are shallower, at any time the extent of moving grains is shorter, and erosion by each avalanche in the quasi-periodic regime is significantly greater. Despite these differences, we observe the same behavior as described above, with intermittency between one regime of quasi-periodic, system-spanning avalanches and another regime of irregular avalanches that can both stop, and overrun and mobilize existing stopped fronts. The duration of each instance of the quasi-periodic regime is reduced to 2-3 avalanches, but behavior in this regime remains clearly distinct from that in the irregular regime, as is shown in Fig. 3. In the irregular regime, the correlation between the avalanche lengths predicted by the overrunning mobilization mechanism and the observed avalanche lengths, using a value of $H$ calculated from 10 previous avalanches, is $0.20\left(p=10^{-14}\right.$ under the assumption that avalanche lengths are random, sampled from their observed distribution). 
The similarity of the system's behavior, despite different properties on the grain and avalanche scales, indicates the applicability of this work to a variety of granular systems. The measured flowability of pharmaceutical granules will differ depending in which of the two regimes the measurement system is in, while alternation between regimes will affect the crossbedding of sedimentary rocks laid down by surface avalanching of grains. Our approach may also be useful in describing disordered systems under shear, where observations of similar intermittency have been made [30-32].

The differences in the system's behavior, meanwhile, indicate the possibility of reconciling the two classes of previous experiments: those that exhibit the power-law size statistics associated with self-organised criticality, and those that instead exhibit quasi-periodic systemspanning avalanches. The shallower avalanches of the smaller glass beads have less inertia than those of sand, so that a smaller degree of slope variability is sufficient for avalanches to stop, and hence for the quasi-periodic regime to be disrupted. This would explain the reduced duration of the quasi-periodic regime. From this perspective, in [16, 17], small rearrangements display power-law statistics because even the slope variability associated with individual grains is sufficient to stop the low inertia motion of small numbers of grains. In [15], one of the varieties of rice has more spherical grains than the other two, and this sphericity not only allows inertia to be higher, due to lower rolling friction, but also reduces the energy barriers associated with the variability of the pile's surface. As a result, avalanches on a small pile of this variety of rice have a narrow size distribution, rather than the broad power-law distribution exhibited by avalanches of the other two varieties. In [22] the system is too small for slope variability to stop avalanches, while in [18-21], the average slope angle is increased after each avalanche by the rotation of the different drums, so that the system is maintained in the quasi-periodic regime. A system such as that we have described, with macroscopic avalanches propagating on a slope both undisturbed by rotation and sufficiently long for avalanches to be able to stop, can support both the quasi-periodic regime and the irregular regime, and will exhibit intermittency between them.

We gratefully acknowledge the technical support of Paul Mitton and David Page-Croft. This work was supported by the National Environmental Research Council [grant number NE/L002507/1] and the Royal Society [grant number DH120121]. 
[1] R. A. Bagnold, The Physics of Blown Sand and Desert Dunes (Methuen, 1941).

[2] S. L. F. Sutton, C. McKenna Neuman, and W. Nickling, Journal of Geophysical Research: Earth Surface 118, 1767 (2013).

[3] D. Kingman, W. Field, and D. Maier, Journal of Agricultural Safety and Health 7, 169 (2001).

[4] J. M. Broder, "Silos Loom as Death Traps on American Farms," (2012), retrieved from www.nytimes.com.

[5] F. Lavoie, L. Cartilier, and R. Thibert, Pharmaceutical Research 19, 887 (2002).

[6] B. Luckman, in The Encyclopedia of Quaternary Science, vol. 3, edited by S. Elias (Elsevier, 2013) pp. pp. 566-573.

[7] M. J. Aschwanden, N. B. Crosby, M. Dimitropoulou, M. K. Georgoulis, S. Hergarten, J. McAteer, A. V. Milovanov, S. Mineshige, L. Morales, N. Nishizuka, G. Pruessner, R. Sanchez, A. S. Sharma, A. Strugarek, and V. Uritsky, Space Science Reviews 198, 47 (2016).

[8] B. Drossel, Phys. Rev. Lett. 76, 936 (1996).

[9] D.-M. Chen, S. Wu, A. Guo, and Z. R. Yang, Journal of Physics A: Mathematical and General 28, 5177 (1995).

[10] G. Grinstein, "Generic scale invariance and self-organized criticality," in Scale Invariance, Interfaces, and Non-Equilibrium Dynamics, edited by A. McKane, M. Droz, J. Vannimenus, and D. Wolf (Springer US, Boston, MA, 1995) pp. 261-293.

[11] R. Frigg, Studies in History and Philosophy of Science Part A 34, 613 (2003).

[12] P. Bak, C. Tang, and K. Wiesenfeld, Phys. Rev. Lett. 59, 381 (1987).

[13] P. Bak, C. Tang, and K. Wiesenfeld, Phys. Rev. A 38, 364 (1988).

[14] L. P. Kadanoff, S. R. Nagel, L. Wu, and S.-m. Zhou, Phys. Rev. A 39, 6524 (1989).

[15] V. Frette, K. Christensen, A. Malthe-Sorenssen, J. Feder, T. Jossang, and P. Meakin, Nature 379, 49 (1996).

[16] M. Bretz, J. B. Cunningham, P. L. Kurczynski, and F. Nori, Phys. Rev. Lett. 69, 2431 (1992).

[17] S. K. de Richter, G. L. Caër, and R. Delannay, Journal of Statistical Mechanics: Theory and Experiment 2012, P04013 (2012).

[18] P. Evesque and J. Rajchenbach, Comptes Rendus de l'Académie des sciences, Série 2 307, p223 (1988). 
[19] H. M. Jaeger, C.-h. Liu, and S. R. Nagel, Phys. Rev. Lett. 62, 40 (1989).

[20] P. Evesque, Phys. Rev. A 43, 2720 (1991).

[21] M. Caponeri, S. Douady, S. Fauve, and C. Laroche, in Mobile Particulate Systems, Vol. 287, edited by E. Guazelli and L. Oger (Kluwer Academic Publishers, 1995) pp. 331-365.

[22] P.-A. Lemieux and D. J. Durian, Phys. Rev. Lett. 85, 4273 (2000).

[23] A. Daerr and S. Douady, Nature 399, 241 (1999), 10.1038/20392.

[24] L. Quartier, B. Andreotti, S. Douady, and A. Daerr, Phys. Rev. E 62, 8299 (2000).

[25] M. G. Kleinhans, H. Markies, S. J. de Vet, A. C. in 't Veld, and F. N. Postema, Journal of Geophysical Research: Planets 116 (2011), 10.1029/2011JE003865.

[26] See Supplemental Material at [URL will be inserted by publisher] for footage of avalanching in each regime.

[27] N. W. Watkins, G. Pruessner, S. C. Chapman, N. B. Crosby, and H. J. Jensen, Space Science Reviews 198, 3 (2016).

[28] T. Börzsönyi, T. C. Halsey, and R. E. Ecke, Phys. Rev. Lett. 94, 208001 (2005).

[29] D. Takagi, J. N. McElwaine, and H. E. Huppert, Phys. Rev. E 83, 031306 (2011).

[30] K. Dahmen, D. Ertas, and Y. Ben-Zion, Phys. Rev. E 58, 1494 (1998).

[31] Y. Ben-Zion, K. Dahmen, V. Lyakhovsky, D. Ertas, and A. Agnon, Earth and Planetary Science Letters 172, 11 (1999).

[32] N. W. Hayman, L. Ducloué, K. L. Foco, and K. E. Daniels, Pure and Applied Geophysics 168, 2239 (2011). 